\title{
Role of RAB5A in FSHR-mediated signal transduction in human granulosa cells
}

\author{
Kai Zhu ${ }^{1,2, *}$, Shang $\mathrm{Li}^{1,2, *}$, Jiansheng Liu' ${ }^{1,2}$, Yan Hong ${ }^{1,2}$, Zi-Jiang Chen ${ }^{1,2,3}$ and Yanzhi Du ${ }^{1,2}$ \\ ${ }^{1}$ Center for Reproductive Medicine, Ren Ji Hospital, School of Medicine, Shanghai Jiao Tong University, Shanghai, \\ China, ${ }^{2}$ Shanghai Key Laboratory for Assisted Reproduction and Reproductive Genetics, Shanghai, China and \\ ${ }^{3}$ National Research Center for Assisted Reproductive Technology and Reproductive Genetics, The Key Laboratory \\ for Reproductive Endocrinology of Ministry of Education, Shandong Provincial Key Laboratory of Reproductive \\ Medicine, Center for Reproductive Medicine, Shandong Provincial Hospital, Shandong University, Jinan, China
} Correspondence should be addressed to Y Du; Email: yanzhidu@hotmail.com

*(K Zhu and S Li contributed equally to this work)

\begin{abstract}
Polycystic ovary syndrome, a common condition characterized by endocrine dysfunction, menstrual irregularity, anovulation and polycystic ovaries, affects $5-7 \%$ of reproductive-age women. $R A B 5 B$, which is identified by a genome-wide association study as a risk locus for this syndrome, encodes a small GTPase involved in control of receptor internalization and early endosome fusion. We found that RAB5A mRNA levels in luteinized granulosa cells of obese patients with polycystic ovary syndrome were lower than in those of obese women without the syndrome. RAB5A regulated follicle-stimulating hormone (FSH)-mediated translocation of the FSH receptor (FSHR) from the membrane to the cytoplasm and the subsequent FSH-FSHR signaling pathway. We showed that RAB5A negatively regulated aromatase expression and estradiol synthesis in human granulosa cells in association with changes in FSHR levels by way of the CAMP/PKA/CREB pathway. The regulation of FSHR by RAB5A may have been associated with two transcription factors, USF1 and USF2. In conclusion, RAB5A gene was abnormally expressed in luteinized granulosa cells of obese patients with polycystic ovary syndrome, which may help explain high FSHR levels found in this syndrome.

Reproduction (2018) 155 505-514
\end{abstract}

\section{Introduction}

Polycystic ovary syndrome (PCOS), a heterogeneous syndrome characterized by endocrine dysfunction, menstrual irregularity, anovulation and polycystic ovaries, is present in $7 \%$ of women of reproductive age (Ehrmann 2005, Mastorakos et al. 2006). The pathogenesis of PCOS is extremely complicated, and its precise etiology remains unclear. A previously conducted genome-wide association study in Han Chinese populations identified several loci associated with a risk for PCOS, including insulin receptor (INSR), follicle-stimulating hormone receptor (FSHR), luteinizing hormone/chorionic gonadotropin receptor (LHCGR), YAP1, C9ORF3, DENND1A, RAB5B, HMGA2, TOX3, SUMO1P1/ZNF217 and THADA. These genes are primarily associated with insulin signaling, hormone function, type 2 diabetes, calcium signaling and endocytosis (Chen et al. 2011, Shi et al. 2012). We hypothesized that the receptor genes in particular may play key roles in the pathogenesis of PCOS.

In response to extracellular stimuli, a ligand-receptor complex is internalized into the cell by endocytosis via the arrestin- and clathrin-mediated pathways. The receptors are subsequently either recycled back to the plasma membrane or transported to lysosomes for degradation (Gong et al. 2008, Jean-Alphonse \& Hanyaloglu 2011). However, evidence increasingly indicates that receptor internalization functions not only in receptor degradation but also in receptor signal transduction (Di Fiore \& De Camilli 2001, Sorkin \& Von Zastrow 2002, Miaczynska et al. 2004, Kang et al. 2005).

Rab proteins are low-molecular-weight guanine nucleotide-binding proteins ( $\mathrm{G}$ proteins) that are located on distinct intracellular membranes and regulate different stages of intracellular membrane trafficking (Pereira-Leal \& Seabra 2001, Schwartz et al. 2007). Rab GTPases are members of the Ras superfamily, in which at least 60 Rabs have been identified. Among these, RAB5A plays a crucial role in clathrin-coated vesicle formation, endosome motility and early endosome fusion (Stenmark 2009). It mediates a cascade of intracellular signaling events by recruiting different effectors to the endosome membrane (Olchowik \& Miaczyńska 2009). RAB5A is required for the interaction of insulin receptor substrate 1 with the p85 subunit of PI3K in NIH3T3 cells, which may be associated with insulin resistance in diabetes 
(Su et al. 2006). Inactive RAB5A (RAB5A: S34N) blocks the epidermal growth factor (EGF)-activated Raf-ERK1/2 kinase pathway and EGF-induced cyclin D1 transcription (Barbieri et al. 2004). In the nucleus, APPL1, an effector of RAB5A, interacts with the nucleosome remodeling and histone deacetylase multiprotein complex, an effect essential for transcriptional regulation of genes regulating cell proliferation (Miaczynska et al. 2004). In response to EGF, RAB5A and its effector APPL1 may translocate from the membrane to the nucleus via an endosomal compartment.

FSHR is a member of G-protein-coupled receptor family comprising a large extracellular domain and a transmembrane domain with seven $\alpha$-helices (Jiang et al. 2012, Menon \& Menon 2012). In testicular Sertoli cells, FSHR is involved in the initiation of spermatogenesis at puberty and maintenance of normal sperm production. In ovarian granulosa cells, it participates in follicular recruitment, growth and eventually maturation (Richards 1980, Simoni et al. 1997). Fshr-deficient male mice have small testes and partial failure of spermatogenesis (Dierich et al. 1998). Fshr-knockout female mice have thin uteri and small ovaries and are sterile because folliculogenesis is blocked before the formation of antral follicles (Balla et al. 2003).

In granulosa cells, FSH binding to FSHR leads to rapid activation of numerous signaling molecules with diverse effects. RAB5A mediates internalization of the $\mathrm{FSH}-$ FSHR complex (Krishnamurthy et al. 2003) but may also participate in FSHR-mediated signal transduction. We found differential $R A B 5 A$ expression in ovarian granulosa cells from obese patients with PCOS compared with obese women without PCOS. Thus, we hypothesize that RAB5A could participate in the dynamic regulation of FSHR localization during folliculogenesis in human ovaries, which could imply involvement in the pathogenesis of PCOS. Further, RAB5A might negatively regulate aromatase expression and estradiol synthesis in association with changes in FSHR levels. This study focused on the function of RAB5A in human granulosa cells (termed hGCs when referring to cells obtained from study participants), attempting to elucidate the molecular mechanisms underlying PCOS.

\section{Materials and methods}

\section{Subjects}

A total of 55 women were recruited from the Center for Reproductive Medicine, Ren Ji Hospital, Shanghai Jiao Tong University School of Medicine. All women gave written informed consent to participate in this study. The study was approved by the assisted reproductive technology (ART) Ethics Committee of Ren Ji Hospital, School of Medicine, Shanghai Jiao Tong University. Twenty-six participants were women 20-35 years old undergoing in vitro fertilization (IVF) and embryo transfer who had been diagnosed with PCOS by meeting at least two or all three of the Rotterdam criteria. The remaining 29 participants were healthy women undergoing IVF for tubal factor infertility or male factor infertility. These 29 women all had regular menstrual cycles (26-35 days) and normal ovarian morphology. Endocrine parameters were measured in this group to exclude hyperandrogenism. Both groups were divided on the basis of body mass index (BMI) into those with normal weight (BMI $18.5-24 \mathrm{~kg} / \mathrm{m}^{2}$ ) and those who were obese $\left(\mathrm{BMI} \geq 24 \mathrm{~kg} / \mathrm{m}^{2}\right.$ ). None of the participants had used hormonal therapy for at least 3 months before the study. All subjects were of Han ethnicity. Each of them underwent a gonadotropin-releasing hormone $(\mathrm{GNRH})$ agonist long protocol consisting of decapeptyl $0.05 \mathrm{mg}$ starting in the luteal phase (menstrual cycle day 21) of the previous cycle until human chorionic gonadotropin (hCG) administration and gonadotropins for 8-12 days after pituitary suppression (i.e. FSH $\leq 5 \mathrm{IU} / \mathrm{L}, \mathrm{LH} \leq 5 \mathrm{IU} / \mathrm{L}$, estradiol $\left(\mathrm{E}_{2}\right) \leq 55 \mathrm{pg} / \mathrm{mL}$, and endometria $<5 \mathrm{~mm}$ ) with an hCG trigger. The patients' clinical information is presented in Supplementary Table 1 (see section on supplementary data given at the end of this article).

Follicular fluid samples were aspirated under ultrasound guidance transvaginally from individual follicles $36 \mathrm{~h}$ after hCG injection. To avoid post-aspiration cell death, the fluid was immediately centrifuged at $201 \boldsymbol{g}$ for $10 \mathrm{~min}$. The cell pellet was resuspended in $0.01 \%$ phosphate-buffered saline (PBS). The hGCs were isolated from the cell suspension with Ficoll gradient centrifugation and then digested with hyaluronidase (Sigma Chemical) for $10 \mathrm{~min}$ at $37^{\circ} \mathrm{C}$. Red blood cells were removed with a $50 \%$ Percoll gradient. The remaining hGCs were purified by centrifugation and then used for extraction of RNA.

\section{Cell culture}

The human granulosa cell line KGN, kindly provided by Shandong University, was also used in our study. KGN cells were maintained in phenol red-free DMEM/F12 (Gibco) supplemented with $10 \%$ fetal bovine serum (Gibco) and were plated at $2 \times 10^{5}$ cells per well in six-well plates and then cultured at $37^{\circ} \mathrm{C}$ in humidified air with $5 \% \mathrm{CO}_{2}$. Cells at passages 5-15 were used in experiments. For FSH stimulation, the culture medium was replaced with medium supplemented with $25 \mathrm{ng} / \mathrm{mL}$ FSH (Merck Serono SA, Aubonne Branch, Switzerland) for $12 \mathrm{~h}$. For the inhibition experiment, we added the protein kinase A (PKA) inhibitor $\mathrm{H}-8910 \mu \mathrm{mol} / \mathrm{L}$ (Selleckchem, Houston, TX, USA) for $30 \mathrm{~min}$.

\section{Immunofluorescence}

KGN cells $\left(2 \times 10^{5}\right)$ were seeded on glass coverslips in six-well cell culture plates overnight and grown to $60 \%$ confluence. Then, the cells were exposed to $400 \mathrm{ng} / \mathrm{mL} \mathrm{FSH}$ for $30 \mathrm{~min}$ at $37^{\circ} \mathrm{C}$. The cells were washed three times with PBS, fixed in $4 \%$ paraformaldehyde for $20 \mathrm{~min}$ at room temperature and permeabilized with $0.2 \%$ Triton X-100 for $20 \mathrm{~min}$. After washing, the samples were blocked with 5\% BSA in PBS for $1 \mathrm{~h}$. To detect the location of FSHR and RAB5A, the cells were incubated with anti-RAB5A (Santa Cruz Biotechnology) at a 1:50 dilution overnight at $4{ }^{\circ} \mathrm{C}$ and then incubated with antiFSHR (Santa Cruz) at a 1:50 dilution at room temperature for 
$2 \mathrm{~h}$. Cells were washed three times with PBS and incubated with AlexaFluor 488 goat anti-rabbit IgG (Proteintech, Wuhan, China) and AlexFluor Cy3 donkey anti-goat IgG (Proteintech) for $2 \mathrm{~h}$ at room temperature. After dyeing with DAPI (Thermo Fisher Scientific), the samples were scanned with a Carl Zeiss inverted fluorescence microscope using a $\times 40$ objective. Carl Zeiss Axio Vision software was used to collect data using a sequential scanning mode to minimize signal cross-over.

\section{Extraction of RNA and analysis with real-time quantitative PCR (RT-qPCR)}

Total RNA was isolated from the subjects' hCGs and KGN cells using the Animal Total RNA Isolation Kit (Foregene, Chengdu, China) according to the manufacturer's instructions and reverse-transcribed to cDNA using a Prime Script RT Master Mix Perfect Real Time kit (TaKaRa). The mRNA levels of target genes were determined with RT-qPCR using the transcribed CDNA and Power SYBR Premix ExTaq (TaKaRa). The primers were obtained from the Primer Bank or designed using Primer3 software. Primer sequences and PCR efficiencies are shown in Table 1 . The relative mRNA levels in each sample were calculated according to a standard curve using serial dilutions of known amounts of specific templates against corresponding cycle threshold values. The RNA levels of target genes were weighted to $A C T B$ as an internal control.

\section{Transfection of siRNA}

siRNA sequences directed against $R A B 5 A, R A B 5 B$ and $R A B 5 C$ were synthesized by Gene Pharma Co., Ltd. (Shanghai, China). Transfection of siRNA $(20 \mathrm{nmol} / \mathrm{L})$ was performed using Lipofectamine RNAiMAX (Invitrogen) according to the manufacturer's instructions. The siRNA sequences specific for RAB5A (5'-AACCAGGAATCAGTGTTGTAG-3), RAB5B (5'-AAGACAGCTATGAACGTGAAT-3), and RAB5C (5'-ACCAACACAGATACATTTGCA-3) were selected on the basis of their potency to inhibit target gene expression.

\section{Western blot analysis}

KGN cells were washed with cold PBS $(50 \mathrm{mM}$ phosphate, $\mathrm{pH} 7.4,100 \mathrm{mM} \mathrm{NaCl}$, and $10 \mathrm{mM} \mathrm{KCl}$ ) and lysed with icecold radioimmunoprecipitation assay lysis buffer (Active Motif, Carlsbad, CA, USA) containing a protease inhibitor cocktail (Roche). Membrane protein and cytoplasmic protein were extracted using a Membrane and Cytoplasmic Protein
Extraction Kit (Sangon Biotech, Shanghai, China) according to the manufacturer's instructions. Equal amounts $(40 \mu \mathrm{g})$ of protein were separated on $8 \%$ SDS polyacrylamide gel and transferred onto a nitrocellulose membrane. After blocking with $5 \%$ BSA in Tris-buffered saline for $1 \mathrm{~h}$, the membranes were incubated overnight at $4{ }^{\circ} \mathrm{C}$ with primary antibodies diluted in 5\% BSA/Tris-buffered saline. Antibodies used included anti-RAB5A (Proteintech) at a dilution of 1:500, anti-FSHR (Proteintech) at 1:500, anti-Aromatase (Abcam) at 1:1000, anti-ATP1A2 (Proteintech) at 1:500, anti-T-cAMPregulatory element-binding protein (CREB) (Cell Signaling) at 1:1000 and anti-P-CREB (Cell Signaling) at 1:1000. The membrane was then incubated with an appropriate secondary antibody conjugated with horseradish peroxidase (Sigma) at room temperature for $1 \mathrm{~h}$. An enhanced chemiluminescent detection system (Millipore) was used to detect bands with peroxidase activity. The bands were visualized using a G-Box iChemi Chemiluminescence image capture system (Syngene, Maryland, USA) and quantitated by Gel-Pro Analyzer image analysis software. Anti-GAPDH (Cell Signaling) and antiATP1A2 were used as internal loading controls for cytoplasmic and membrane proteins, respectively.

\section{Transfection of recombinant EGFP-RAB5A: WT}

A full-length human RAB5A cDNA was amplified by PCR and then digested and inserted into EGFP-N1 green fluorescent protein (GFP) to construct a recombinant EGFP-RAB5A: WT. The vectors were transfected with DNA Transfection Reagent (Biotool, Selleckchem, Houston, TX, USA). One day before transfection, $2 \times 10^{5} \mathrm{KGN}$ cells were plated per well in six-well plates. DNA, $4 \mu \mathrm{g}$, and transfection reagent, $10 \mu \mathrm{L}$, were each diluted in $200 \mu \mathrm{L}$ and incubated for $5 \mathrm{~min}$ at room temperature. They were then combined and incubated for $20 \mathrm{~min}$, and $400 \mu \mathrm{L}$ of the mixture was added to each well. The medium was changed $6 \mathrm{~h}$ after transfection. The cells were incubated at $37^{\circ} \mathrm{C}$ in humidified air with $5 \% \mathrm{CO}_{2}$ for $48 \mathrm{~h}$ prior to testing for $R A B 5 A$ gene expression.

\section{Steroid assays}

Testosterone $\left(1 \times 10^{-7} \mathrm{ng} / \mathrm{mL}\right)$ (Sigma) was added as the substrate for $E_{2}$ synthesis. After $4 h$, the levels of $E_{2}$ in the spent media were measured using an electrochemiluminescence immunoassay (ECLIA) kit (Roche) according to the manufacturer's instructions. The levels of $E_{2}$ in the peripheral blood of recruited subjects were also detected by ECLIA.

Table 1 Primer sequences used for RT-qPCR and PCR efficiencies.

\begin{tabular}{llc}
\hline \multirow{2}{*}{ Gene } & \multicolumn{2}{c}{ Primer sequence $\left(5^{\prime}-3^{\prime}\right)$} \\
\cline { 2 - 3 } ACTB & Forward & Reverse \\
FSHR & GGGAAATCGTGCGTGACATTAAG & TGTGTTGGCGTACAGGTCTTTG \\
CYP19A1 & TCTGTCACTGCTCTAACAGGG & TGCACCTTTTGGATGACTCG \\
RAB5A & TGGAAATGCTGAACCCGATAC & AATTCCCATGCAGTAGCCAGG \\
RAB5B & AGACCCAACGGGCCAAATAC & GCCCCAATGGTACTCTCTTGAA \\
RAB5C & TGGCTACCCAGTGAAAAAGG & CCATGGCGATGTACTTTCCT \\
USF1 & CCGCTTTGTCAAGGGACAGTT & AGGCTGTGATACCGCTCCT \\
USF2 & CTGCTGTTGTTACTACCCAGG & TCTGACTTCGGGGAATAAGGG \\
\hline
\end{tabular}




\section{Statistical analysis}

After inspection for normal distribution of the data, differences between groups were determined using a one-way ANOVA test followed by the Newman-Keuls multiple comparison test. Multiple group comparisons were performed with a twoway ANOVA test followed by the Bonferroni post-test where appropriate to assess significant differences. Correlations between groups were analyzed by Pearson's correlation coefficient. Data analyses were conducted using SPSS 21.0 for Windows (IBM) and GraphPad Prism, version 5.01 (GraphPad). $P$ values $<0.05$ were considered statistically significant.

\section{Results}

RAB5A is abnormally expressed in granulosa cells of patients with PCOS

To examine whether $R A B 5 A, R A B 5 B$ and $R A B 5 C$ were differentially expressed in PCOS and non-PCOS granulosa cells, we collected hGCs from 55 women undergoing IVF with a GNRHa long protocol, 26 with and 29 without PCOS. We also compared results of the normal group $\left(18.5 \mathrm{~kg} / \mathrm{m}^{2} \leq \mathrm{BMl}<24 \mathrm{~kg} / \mathrm{m}^{2}\right)$ vs those in the obese group (BMI $\geq 24 \mathrm{~kg} / \mathrm{m}^{2}$ ). RAB5A mRNA levels in hGCs from patients with PCOS were lower than in those without PCOS, but this difference was observed only among the women who were obese. There were no statistically significant differences in $R A B 5 B$ and $R A B 5 C$ expression (Fig. 1).

\section{RAB5A mediated FSH-induced internalization of FSHR}

We found both FSHR and RAB5A were localized on the KGN cell membrane (Fig. 2). With FSH stimulation, FSH ligand-receptor complexes were endocytosed, as indicated by reduced amounts of FSHR on the cell membrane and increased levels in the cytoplasm. RAB5A underwent a similar dramatic change. This simultaneous subcellular localization of FSHR and RAB5A implies in part that RAB5A was involved in the $\mathrm{FSH}$-induced internalization of FSHR.

We examined FSHR levels in the cytoplasm and on the membrane by Western blotting (Fig. 3). In the absence of FSH treatment, FSHR was mainly located on the cell membrane. After FSH stimulation, FSHR underwent internalization, indicated by the finding that, following interaction with its ligand, FSHR expression on the membrane decreased and that in the cytoplasm increased. That is, the ratio of FSHR on the membrane to that in the cytoplasm in treated cells was lower than the ratio in negative control cells. However, in the RAB5A siRNA-transfected cells, there was no significant reduction of FSHR on the membrane after $\mathrm{FSH}$ stimulation. These results suggest that downregulation of RAB5A prevents FSH-induced internalization of FSHR.

\section{RAB5A expression affected the total FSHR levels in KGN cells}

We tested FSHR levels in KGN cells after knockdown of $R A B 5 A$. We found that FSHR levels increased after FSH treatment both in the NC group and in the RAB5A siRNA-transfected group in a similar manner. Higher FSHR protein and mRNA levels were observed in KGN cells after RAB5A knockdown in the presence or absence of FSH (Fig. 4A and B). Treatment of cells with siRNA targeting RAB5B or RAB5C had no significant effect on FSHR mRNA levels (Supplementary Fig. 1A). To further study the role of RAB5A in FSHR expression, we overexpressed RAB5A in KGN cells (Fig. 4C). Addition of FSH increased FSHR protein levels in GFP controls but had no effect in cells overexpressing RAB5A (Fig. 4D). FSHR mRNA levels were reduced in RAB5A-transfected cells in the presence of FSH (Fig. 4E).

The basal helix-loop-helix transcription factors upstream stimulatory factor (USF) 1 and USF2, two transcription factors, which bind to the E-box of the FSHR promoter, play key roles in regulating FSHR transcriptional activity (Heckert et al. 1998, 2000). We found that downregulation of RAB5A increased USF1 and USF2 mRNA levels (Supplementary Fig. 1B), and RAB5A upregulation decreased USF1 and USF2 mRNA levels (Supplementary Fig. 1C). Therefore, the regulation of FSHR by RAB5A was probably related to USF1 and USF2.

\section{$R A B 5 A$ regulated aromatase expression and estradiol synthesis in KGN cells}

In granulosa cells, the FSH signaling cascade has diverse effects that are crucial for human fertility. One primary
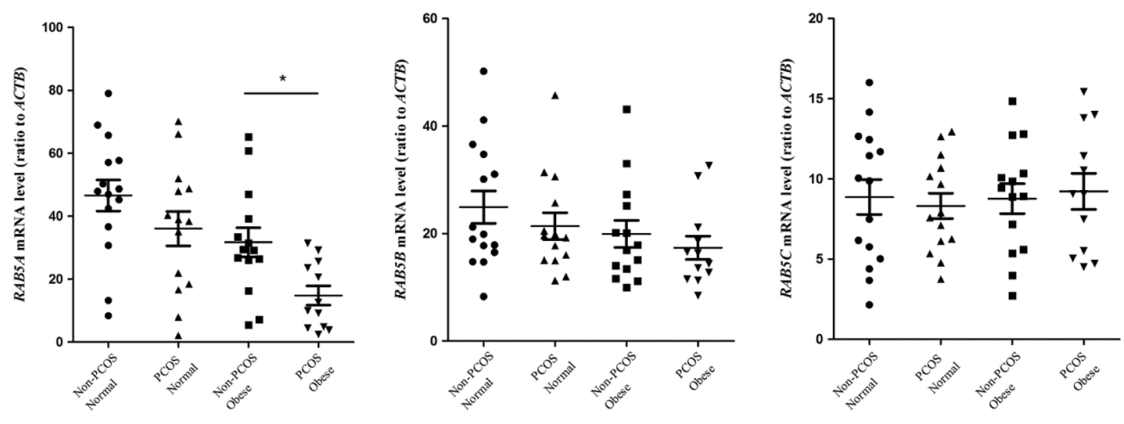

Figure 1 Decreased expression of $R A B 5 A$ in ovarian granulosa cells of patients with polycystic ovary syndrome (PCOS). RAB5A, $R A B 5 B$ and $R A B 5 C$ mRNA levels detected by RT-qPCR in ovarian granulosa cells from women who were undergoing in vitro fertilization, 26 with and 29 without PCOS. Data are presented as mean \pm S.E.M. $* P<0.05$. 

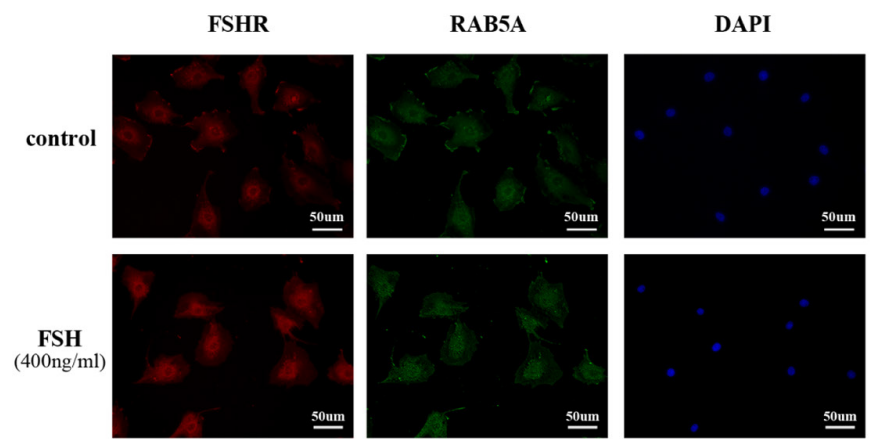

and well-characterized effect is the secretion of $E_{2}$, which is synthesized by an aromatase enzyme encoded by the CYP19A1 gene. Our study showed that CYP19A1 mRNA, aromatase protein and $\mathrm{E}_{2}$ levels all increased in KGN cells stimulated by FSH $(25 \mathrm{ng} / \mathrm{mL})$ for $12 \mathrm{~h}$. RAB5A knockdown increased both basal and FSH-stimulated CYP19A1 and aromatase levels as well as $\mathrm{E}_{2}$ levels in KGN cells. There was no difference in the extent of aromatase increase after FSH treatment (Fig. 5A, B and C). On the other hand, in cells transfected with either an empty vector or with a recombinant $R A B 5 A$ vector, there was an increase in CYP19A1 mRNA levels. However, aromatase levels increased only in the cells transfected with the empty vector. RAB5A overexpression was associated with decreases only in FSH-stimulated CYP19A1 mRNA and aromatase protein levels (Fig. 5D and E). Compared with $R A B 5 A, R A B 5 B$ and $R A B 5 C$ had similar although smaller effects in regard to regulation of CYP19A1 expression and $\mathrm{E}_{2}$ synthesis (Supplementary Fig. 2B and C).

\section{The role of $R A B 5 A$ in the activation of $C A M P / P K A$ / CREB pathway}

Of the many signaling pathways regulating aromatase activity, the cAMP/PKA/CREB pathway is the primary one. Upon FSH stimulation, FSH combines with its receptor, activating adenylyl cyclase, which results in increased cyclic AMP (cAMP) production (Richards et al. 1979). CAMP, in turn, activates AMP-dependent PKA, leading to

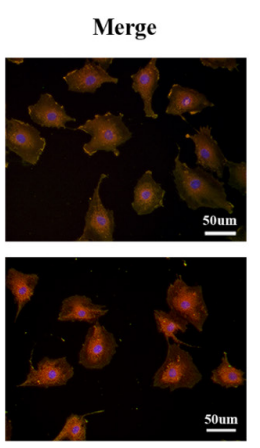

Figure 2 Localization of FSHR and RAB5A in human granulosa cells. Expression and localization of FSHR (red signal) and RAB5A (green signal) in KGN cells detected by immunofluorescence assay. Cells were treated with medium alone (control group) or with FSH (400 ng/mL) (stimulation group).

the phosphorylation of CREB (Walker et al. 1995). CREB then binds to the CYP19A1 promoter region to facilitate the gene's transcription (Mendelson \& Kamat 2007). To explore the molecular mechanism of RAB5A in the regulation of aromatase expression, Western blotting was used to assess activation of CAMP/PKA/CREB pathway in FSH-stimulated KGN cells with or without $R A B 5 A$ knockdown. FSH induced phosphorylation of CREB at Ser 133, reaching a maximum when the cells were treated with FSH for $1 \mathrm{~h}$. The degree of CREB phosphorylation in cells transfected with RAB5A siRNA was greater than that in cells transfected with NC siRNA. Maximum phosphorylation was also achieved after $1 \mathrm{~h}$ of $\mathrm{FSH}$ treatment in $R A B 5 A$-downregulated KGN cells. Therefore, RAB5A knockdown did not increase the speed of CREB phosphorylation (Fig. 6A). KGN cells were incubated with $10 \mu \mathrm{mol} / \mathrm{L}$ of the PKA inhibitor $\mathrm{H}-89$ for 30 min before FSH treatment. H-89 decreased $E_{2}$ and aromatase protein levels in both the NC group and the RAB5A siRNA-transfected group. Similarly, the increased expression of $\mathrm{E}_{2}$ and aromatase induced by $R A B 5 A$ knockdown was also blocked by $\mathrm{H}-89$ (Fig. 6B and C). Conversely, upregulation of RAB5A weakened $\mathrm{FSH}$-induced CREB phosphorylation. CREB was phosphorylated after $1 \mathrm{~h}$ of FSH stimulation in KGN cells transfected with an empty vector but not in cells with high RAB5A expression (Fig. 6D). These results demonstrate that $\mathrm{RAB} 5 \mathrm{~A}$ participates in the regulation of aromatase expression in part by way of the CAMP/PKA/CREB pathway.

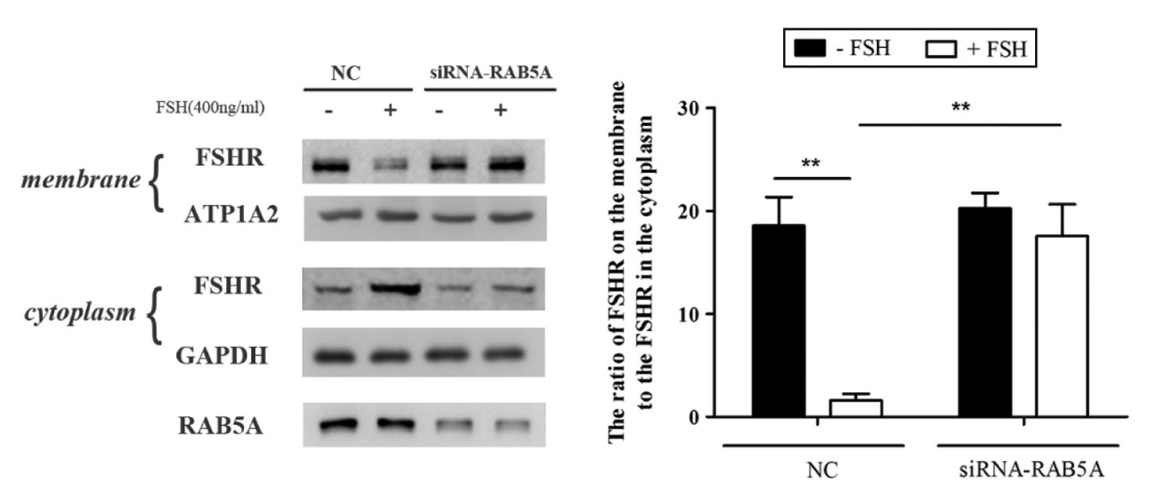

Figure 3 RAB5A mediated FSH-induced internalization of the FSHR. FSHR protein levels detected by Western blot in the cytoplasm or cell membrane after RAB5A knockdown in KGN cells. GAPDH was used as the reference gene for the cytoplasm and ATPase $\mathrm{Na}+/ \mathrm{K}+$ transporting subunit alpha 2 (ATP1A2) as the reference gene for the cell membrane. The ratio of FSHR on the membrane to that in the cytoplasm was calculated by the numerical values normalized with their respective references. The blots are representative of assays performed in triplicate. ${ }^{* *} P<0.01$. 
A
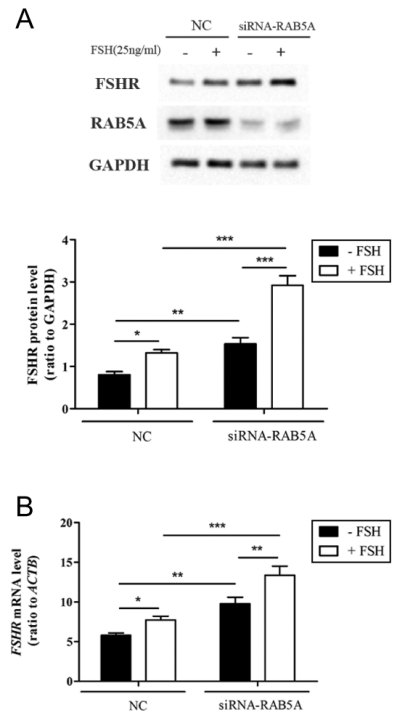

C

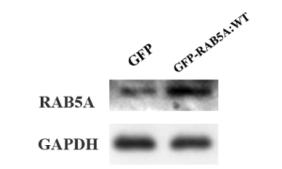

D
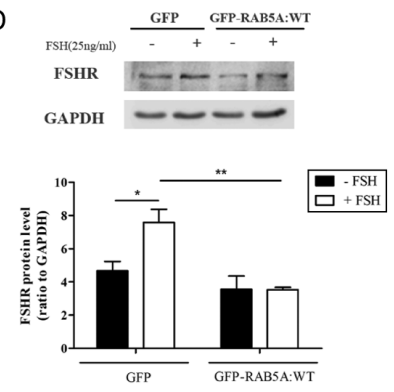

E

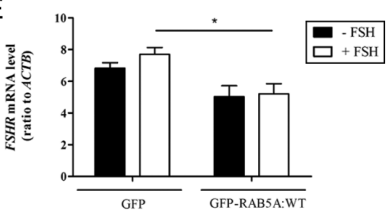

Figure 4 RAB5A affected the total level of FSHR in KGN cells. (A) FSHR protein levels detected by Western blot analysis after RAB5A knockdown in KGN cells with or without FSH $(25 \mathrm{ng} / \mathrm{mL})$ stimulation for $12 \mathrm{~h}$. Above, a representative image is shown. Below, the immunoreactive bands are densitometrically quantified. (B) FSHR mRNA levels detected by RT-qPCR after RAB5A knockdown with or without $\mathrm{FSH}(25 \mathrm{ng} / \mathrm{mL})$ stimulation for $12 \mathrm{~h}$. (C) A representative image of an $R A B 5 A$ construct after an $R A B 5 A$ recombinant was transfected into KGN cells is shown. (D) FSHR protein levels after RAB5A overexpression detected by Western blot in KGN cells with or without FSH $(25 \mathrm{ng} / \mathrm{mL})$ stimulation for $12 \mathrm{~h}$. Above, a representative image is shown. Below, the immunoreactive bands are densitometrically quantified. (E) FSHR mRNA levels detected by RT-qPCR after $R A B 5 A$ overexpression with or without $\mathrm{FSH}(25 \mathrm{ng} / \mathrm{mL})$ stimulation for $12 \mathrm{~h}$. Data are means \pm S.E.M. from four experiments. ${ }^{*} P<0.05,{ }^{* *} P<0.01,{ }^{* * *} P<0.001$.

\section{The negative correlation between RAB5A expression and $E_{2}$ levels in patients undergoing IVF}

To further study the relationship between RAB5A mRNA expression and $E_{2}$ levels, we tested hGCs from patients with or without PCOS who were undergoing the same IVF protocol. We assessed the correlation between RAB5A mRNA levels in primary hGCs of patients with or without PCOS and peripheral blood $\mathrm{E}_{2}$ levels on the third day of the menstrual cycle. RAB5A mRNA levels in hGCs were negatively correlated with $\mathrm{E}_{2}$ levels in both groups (Fig. 7).

\section{Discussion}

RAB5A, a small GTPase protein, mediates vital signaling transduction by recruiting several RAB5A effectors, such as PI3Ks, rabaptin-5, EEA-1 and rabenosyn-5, on the endosome membrane (Stenmark et al. 1995, Christoforidis et al. 1999, Nielsen et al. 2000, Dou et al. 2013). Increasing evidence confirms that RAB5A

A
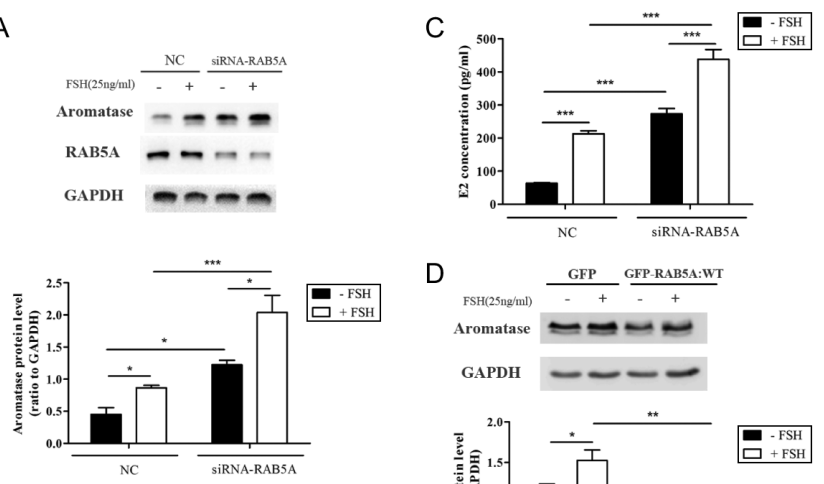

D
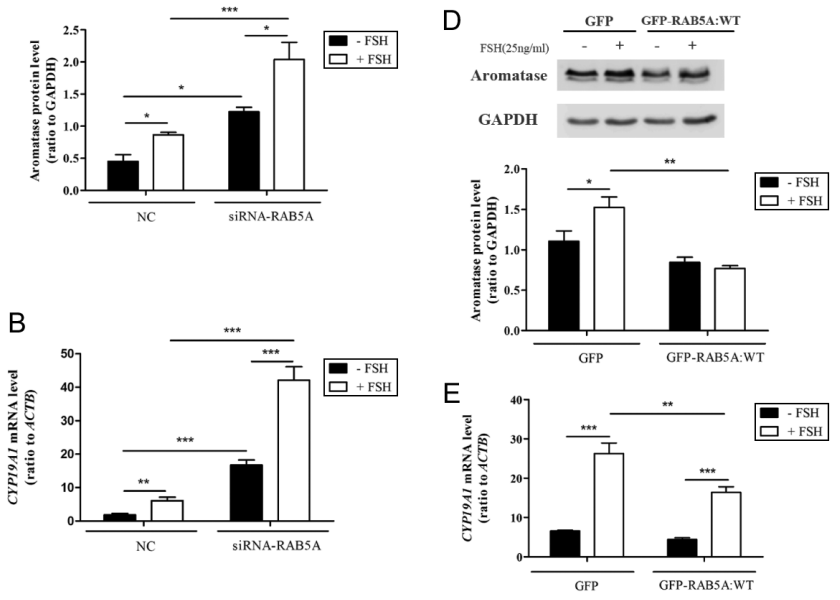

Figure 5 RAB5A regulated aromatase expression and $E_{2}$ synthesis in KGN cells. (A) Aromatase protein levels detected by Western blot after RAB5A knockdown in KGN cells with or without FSH $(25 \mathrm{ng} / \mathrm{mL})$ stimulation for $12 \mathrm{~h}$. Above, a representative image is shown. Below, the immunoreactive bands are densitometrically quantified. (B) CYP19A1mRNA levels detected by RT-qPCR after RAB5A knockdown with or without FSH $(25 \mathrm{ng} / \mathrm{mL})$ stimulation for $12 \mathrm{~h}$. (C) $\mathrm{E}_{2}$ levels in the cell culture supernatant measured after $R A B 5 A$ knockdown and further incubation with FSH. (D) Aromatase protein levels detected by Western blot after RAB5A overexpression in KGN cells with or without FSH ( $25 \mathrm{ng} / \mathrm{mL})$ stimulation for $12 \mathrm{~h}$. Above, a representative Western blot is shown. Below, the immunoreactive bands are densitometrically quantified. (E) CYP19A1 mRNA levels detected by RT-qPCR after RAB5A overexpression with or without FSH stimulation for $12 \mathrm{~h}$. Data are means \pm S.E.M. from three independent experiments. ${ }^{*} P<0.05,{ }^{* *} P<0.01,{ }^{* * *} P<0.001$.

is essential for the maintenance of normal reproductive function. In mammals, the initiation and maintenance of gametogenesis depends not only on the rhythmic variation of sex hormones but also on the dynamic expression of hormone receptors on the germ cell membrane. RAB5A plays an indispensable role in the dynamic recycling of hormone receptors between the cell membrane and cytoplasm (Segretain et al. 2010). RAB5A-regulated transport of the $\mathrm{LH}$ receptor from the cell surface and its subsequent lysosomal degradation may be associated with dynamic regulation of LH receptor expression during the ovarian cycle by permitting the entry of newly synthesized receptors to the cell surface (Gulappa et al. 2011).

In this study, we tested the critical role of RAB5A in FSHR-mediated signal transduction in the human granulosa KGN cell line. The activated receptor complex is reportedly internalized into endosomes after FSH binds to FSHR (Krishnamurthy et al. 2003). We found that downregulation of $R A B 5 A$ blocked internalization 

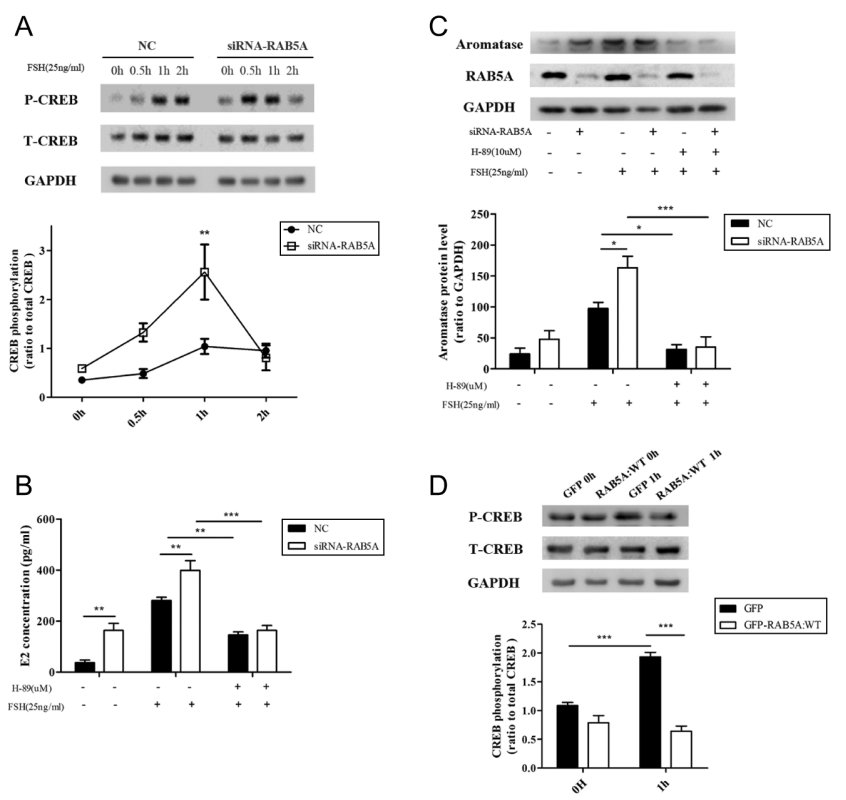

Figure 6 RAB5A regulated aromatase and $E_{2}$ levels via the CAMP/ PKA/CREB pathway. (A) Levels of phosphorylated CREB (P-CREB) and total CREB (T-CREB) after RAB5A knockdown in KGN cells detected at different time points by Western blot analysis. Above, a representative Western blot is shown. Below, the immunoreactive bands of CREB phosphorylation are densitometrically quantified. (B) $\mathrm{E}_{2}$ levels in the cell culture supernatant measured after $R A B 5 \mathrm{~A}$ knockdown and incubation with $10 \mu \mathrm{mol} / \mathrm{L} \mathrm{H}-89$ (PKA inhibitor) for $30 \mathrm{~min}$ and subsequently with $25 \mathrm{ng} / \mathrm{mL} \mathrm{FSH}$ for $12 \mathrm{~h}$. (C) Aromatase and RAB5A protein levels detected by Western blot after RAB5A knockdown and incubation with $10 \mu \mathrm{mol} / \mathrm{L} \mathrm{H}-89$ (PKA inhibitor) for $30 \mathrm{~min}$ and subsequently with $25 \mathrm{ng} / \mathrm{mL} \mathrm{FSH}$ for $12 \mathrm{~h}$. A representative Western blot image is shown. (D) P-CREB and T-CREB protein levels detected by Western blot after RAB5A overexpression in KGN cells. Above, a representative Western blot is shown. Below, the immunoreactive bands are densitometrically quantified. Data are means \pm S.E.M. from three independent experiments. ${ }^{*} P<0.05$, ${ }^{* *} P<0.01,{ }^{* * *} P<0.001$.

of the receptor, as indicated by an increase in FSHR on the membrane. Meanwhile, RAB5A downregulation also increased FSHR expression with or without FSH stimulation. Previous studies have shown a double effect of FSH on FSHR expression. In the absence of FSH or
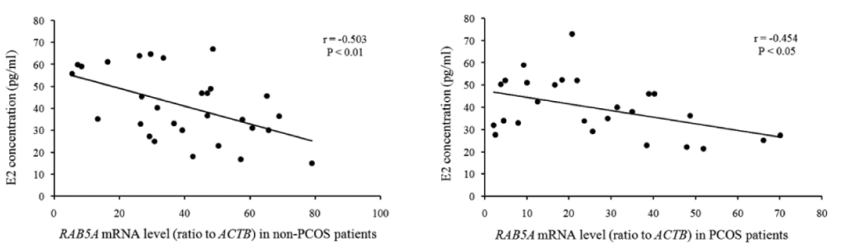

Figure 7 Negative correlation between $R A B 5 A$ expression and $\mathrm{E}_{2}$ levels in patients undergoing in vitro fertilization. Relationship between RAB5A mRNA levels in primary human granulosa cells and peripheral blood $E_{2}$ levels on day 3 of the menstrual cycle of 26 patients with (left) and 29 without (right) polycystic ovary syndrome. The results are based on the calculated Pearson correlation $r$ and $P$ values according to distribution of the relevant variables. a cAMP analog, granulosa cells lose FSHR expression, whereas the addition of FSH or CAMP analog prevents this loss (Galway et al. 1990). In preovulatory granulosa cells, FSH stimulation increases both FSHR mRNA levels and the receptors' binding ability. In contrast, the concentration of FSH during ovulation decreases FSHR mRNA levels and binding ability and then promotes granulosa cell luteinization. In an in vitro experiment, a certain concentration of FSH promoted FSHR expression in luteinized granulosa cells, but when the dose was high enough, FSHR no longer increased (Galway et al. 1990, LaPolt et al. 1992). In our study, we found that $25 \mathrm{ng} /$ $\mathrm{mL}$ of FSH significantly increased FSHR expression in KGN cells. After RAB5A knockdown, FSH may also promote FSHR expression. Therefore, we postulated that the interaction between RAB5A and FSH affected the expression of FSHR. However, RAB5A downregulation did not enhance the effect of FSH on FSHR.

RAB5A downregulation weakens a number of receptor-mediated signaling pathways (Barbieri et al. 2004, Su et al. 2006). In our study, we found that $R A B 5 A$ knockdown increased the phosphorylation of CREB, strengthened FSH-stimulated activation of the CAMP/PKA/CREB pathway in KGN cells and increased aromatase expression and $E_{2}$ synthesis. FSHR cannot be activated without ligand binding. However, we demonstrated that $R A B 5 A$ knockdown increased aromatase levels in the absence of FSH stimulation. Previous evidence indicated a crucial role of RAB5A in the regulation of autophagy, with low RAB5A expression levels prevents autophagy (Su et al. 2011, Marlin \& Li 2015). It has also been reported that autophagy affects aromatase expression by changing its distribution in microsomes and lysosomes (Zhang et al. 2010). Thus, the negative effect of RAB5A on aromatase expression might be related to the inhibition of autophagy. In order to further demonstrate the role of RAB5A in the regulation of $E_{2}$ synthesis, we assessed the correlation between RAB5A expression and $E_{2}$ levels. We found that the RAB5A mRNA levels in hGCs were negatively correlated with peripheral blood $E_{2}$ levels on the third day of the menstrual cycle. Although the pituitary was almost completely suppressed by the GNRH agonist on the third day of the cycle and $E_{2}$ production was mainly derived from the basal ovarian secretory function, $E_{2}$ blood levels are not totally representative of hGC $E_{2}$ synthesis because of hepatic $E_{2}$ degradation. We therefore hypothesized a relationship between RAB5A and $\mathrm{E}_{2}$ synthesis.

$R A B 5 A, R A B 5 B$ and $R A B 5 C$ share more than $90 \%$ sequence identity, but the genes have different functions. Firstly, they are phosphorylated by different kinases in vitro (Chiariello et al. 1999, Chen et al. 2009). RAB5A, but not RAB5B or RAB5C, plays a critical role in early phagosome-endosome interactions and governs the maturation of the early phagosome, leading to phagosome-lysosome fusion (Alvarez-Dominguez 
\& Stahl 1999). Secondly, RAB5A, RAB5B and RAB5C function differently in EGF-induced cell motility. RAB5C, but not RAB5A or RAB5B, is selectively associated with growth factor activation of RAC1 and with enhanced cell motility (Chen et al. 2014). Moreover, RAB5A, RAB5B and $R A B 5 C$ differentially regulate the trafficking and degradation of the EGF receptor (EGFR). Suppression of RAB5A or RAB5B hampers EGFR degradation, whereas RAB5C depletion has very little effect (Chen et al. 2009). We found that RAB5A, RAB5B and RAB5C might have different roles in FSHR-mediated signal transduction in KGN cells. We knocked down the three genes with their specific siRNA sequences. Their low expression increased aromatase expression, but $R A B 5 A$ had the most obvious effect, whereas that of $R A B 5 B$ and $R A B 5 C$ was much lower.

In this study, we used KGN cells as a model for investigating FSHR signaling. Because the KGN cell line was established from a transformed granulosa cell tumor, it does not authentically replicate the physiological environment of the human body. However, it is more likely to be closer to normal physiological conditions than other human cell lines, which, it has been speculated, lost native FSHR upon cell transformation (Nishi et al. 2001).

Previous research showed an increased testosteroneto-estrogen ratio in the follicular fluid of patients with PCOS, implying decreased aromatase expression in granulosa cells in PCOS (Jakimiuk et al. 1998). $E_{2}$ blood levels are also slightly lower in those with PCOS than those without PCOS (Amato et al. 2003). We found that $R A B 5 A$ expression was reduced in hGCs from obese women with PCOS and confirmed that the low RAB5A expression level promoted aromatase expression, which seemed somewhat contradictory. But other studies have suggested that decreased aromatase expression in PCOS is due not only to granulosa cell dysfunction but also to an FSH deficiency and decreased conversion of androgens to estrogen (Hillier 1994, la Marca et al. 2002). In fact, granulosa cells from patients with PCOS have been demonstrated to have increased aromatase activity and $\mathrm{E}_{2}$ synthesis in response to $\mathrm{FSH}$ stimulation (Araki et al. 1996). Other studies have indicated that aromatase activity is related to $\mathrm{FSH}$ levels and the hormone's capacity to combine with its receptor (Franks et al. 2000, 2008). In order to offset an FSH deficiency, feedback may induce increased FSHR levels in granulosa cells from patients with PCOS. This would enhance the sensitivity of FSH-FSHR binding, thereby promoting the conversion of androgens to estrogens. In this study, we found that RAB5A not only regulated FSHmediated translocation of FSHR from the membrane to the cytoplasm of granulosa cells but also mediated the FSHR expression level, which affected activation of the CAMP/PKA/CREB pathway. In conclusion, our study focused on the function of PCOS-associated genes and might provide further insight into the molecular mechanisms involved in the pathogenesis of PCOS.

\section{Supplementary data}

This is linked to the online version of the paper at https://doi.org/10.1530/REP-18-0015.

\section{Declaration of interest}

The authors declare that there is no conflict of interest that could be perceived as prejudicing the impartiality of the research reported.

\section{Funding}

This research was supported by grants from the National Natural Science Foundation (81490743, 81671413 and 81671414), Shanghai Municipal Education Commission Gaofeng Clinical Medicine (20152510), and Shanghai Key Laboratory for Assisted Reproduction and Reproductive Genetics (17DZ2271100).

\section{Acknowledgments}

The authors thank all sample donors and the clinicians and embryologists of the Reproduction Center of Ren Ji Hospital for their excellent assistance.

\section{References}

Alvarez-Dominguez C \& Stahl PD 1999 Increased expression of Rab5a correlates directly with accelerated maturation of Listeria monocytogenes phagosomes. Journal of Biological Chemistry 274 11459-11462. (https:// doi.org/10.1074/jbc.274.17.11459)

Amato G, Conte M, Mazziotti G, Lalli E, Vitolo G, Tucker AT, Bellastella A, Carella C \& Izzo A 2003 Serum and follicular fluid cytokines in polycystic ovary syndrome during stimulated cycles. Obstetrics and Gynecology 101 1177-1182. (https://doi.org/10.1097/00006250200306000-00009)

Araki H, Tsubota T, Maeda N, Harada N, Kominami S, Mason JI \& Kita I 1996 Intraovarian immunolocalization of steroidogenic enzymes in a Hokkaido brown bear, Ursus arctos yesoensis during the mating season. Journal of Veterinary Medical Science 58 787-790. (https://doi. org/10.1292/jvms.58.787)

Balla A, Danilovich N, Yang Y \& Sairam MR 2003 Dynamics of ovarian development in the FORKO immature mouse: structural and functional implications for ovarian reserve. Biology of Reproduction 69 1281-1293. (https://doi.org/10.1095/biolreprod.103.015552)

Barbieri MA, Fernandez-Pol S, Hunker C, Horazdovsky BH \& StahI PD 2004 Role of rab5 in EGF receptor-mediated signal transduction. European Journal of Cell Biology 83 305-314. (https://doi.org/10.1078/0171-933500381)

Chen PI, Kong C, Su X \& Stahl PD 2009 Rab5 isoforms differentially regulate the trafficking and degradation of epidermal growth factor receptors. Journal of Biological Chemistry 284 30328-30338. (https:// doi.org/10.1074/jbc.M109.034546)

Chen ZJ, Zhao H, He L, Shi Y, Qin Y, Shi Y, Li Z, You L, Zhao J, Liu J et al. 2011 Genome-wide association study identifies susceptibility loci for polycystic ovary syndrome on chromosome 2p16.3, 2p21 and 9q33.3. Nature Genetics 43 55-59. (https://doi.org/10.1038/ng.732) 
Chen PI, Schauer K, Kong C, Harding AR, Goud B \& Stahl PD 2014 Rab5 isoforms orchestrate a 'division of labor' in the endocytic network; Rab5C modulates Rac-mediated cell motility. PLOS ONE 9 e90384. (https://doi.org/10.1371/journal.pone.0090384)

Chiariello M, Bruni CB \& Bucci C 1999 The small GTPases Rab5a, Rab5b and Rab5c are differentially phosphorylated in vitro. FEBS Letters 453 20-24. (https://doi.org/10.1016/S0014-5793(99)00686-9)

Christoforidis S, McBride HM, Burgoyne RD \& Zerial M 1999 The Rab5 effector EEA1 is a core component of endosome docking. Nature 397 621-625. (https://doi.org/10.1038/17618)

Di Fiore PP \& De Camilli P 2001 Endocytosis and signaling. An inseparable partnership. Cell 106 1-4. (https://doi.org/10.1016/S00928674(01)00428-7)

Dierich A, Sairam MR, Monaco L, Fimia GM, Gansmuller A, LeMeur M \& Sassone-Corsi P 1998 Impairing follicle-stimulating hormone (FSH) signaling in vivo: targeted disruption of the $\mathrm{FSH}$ receptor leads to aberrant gametogenesis and hormonal imbalance. PNAS 95 13612-13617. (https://doi.org/10.1073/pnas.95.23.13612)

Dou Z, Pan JA, Dbouk HA, Ballou LM, DeLeon JL, Fan Y, Chen JS, Liang Z, Li G, Backer JM, Lin RZ et al. 2013 Class IA PI3K p110beta subunit promotes autophagy through Rab5 small GTPase in response to growth factor limitation. Molecular Cell 50 29-42. (https://doi.org/10.1016/j. molcel.2013.01.022)

Ehrmann DA 2005 Polycystic ovary syndrome. New England Journal of Medicine 352 1223-1236. (https://doi.org/10.1056/NEJMra041536)

Franks S, Mason H \& Willis D 2000 Follicular dynamics in the polycystic ovary syndrome. Molecular and Cellular Endocrinology 163 49-52. (https://doi.org/10.1016/S0303-7207(99)00239-7)

Franks S, Stark J \& Hardy K 2008 Follicle dynamics and anovulation in polycystic ovary syndrome. Human Reproduction Update 14 367-378. (https://doi.org/10.1093/humupd/dmn015)

Galway AB, Lapolt PS, Tsafriri A, Dargan CM, Boime I \& Hsueh AJ 1990 Recombinant follicle-stimulating hormone induces ovulation and tissue plasminogen activator expression in hypophysectomized rats. Endocrinology 127 3023-3028. (https://doi.org/10.1210/endo-127-63023)

Gong Q, Huntsman C \& Ma D 2008 Clathrin-independent internalization and recycling. Journal of Cellular and Molecular Medicine 12 126-144. (https://doi.org/10.1111/j.1582-4934.2007.00148.x)

Gulappa T, Clouser CL \& Menon KM 2011 The role of Rab5a GTPase in endocytosis and post-endocytic trafficking of the hCG-human luteinizing hormone receptor complex. Cellular and Molecular Life Sciences 68 2785-2795. (https://doi.org/10.1007/s00018-010-0594-1)

Heckert LL, Daggett MA \& Chen J 1998 Multiple promoter elements contribute to activity of the follicle-stimulating hormone receptor (FSHR) gene in testicular Sertoli cells. Molecular Endocrinology 12 1499-1512. (https://doi.org/10.1210/mend.12.10.0183)

Heckert LL, Sawadogo M, Daggett MA \& Chen JK 2000 The USF proteins regulate transcription of the follicle-stimulating hormone receptor but are insufficient for cell-specific expression. Molecular Endocrinology 14 1836-1848. (https://doi.org/10.1210/mend.14.11.0557)

Hillier SG 1994 Current concepts of the roles of follicle stimulating hormone and luteinizing hormone in folliculogenesis. Human Reproduction 9 188-191. (https://doi.org/10.1093/oxfordjournals. humrep.a138480)

Jakimiuk AJ, Weitsman SR, Brzechffa PR \& Magoffin DA 1998 Aromatase mRNA expression in individual follicles from polycystic ovaries. Molecular Human Reproduction 4 1-8. (https://doi.org/10.1093/ molehr/4.1.1)

Jean-Alphonse F \& Hanyaloglu AC 2011 Regulation of GPCR signal networks via membrane trafficking. Molecular and Cellular Endocrinology 331 205-214. (https://doi.org/10.1016/j.mce.2010.07.010)

Jiang X, Liu H, Chen X, Chen PH, Fischer D, Sriraman V, Yu HN, Arkinstall S \& He X 2012 Structure of follicle-stimulating hormone in complex with the entire ectodomain of its receptor. PNAS 109 12491-12496. (https:// doi.org/10.1073/pnas.1206643109)

Kang J, Shi Y, Xiang B, Qu B, Su W, Zhu M, Zhang M, Bao G, Wang F, Zhang $\mathbf{X}$ et al. 2005 A nuclear function of beta-arrestin1 in GPCR signaling: regulation of histone acetylation and gene transcription. Cell 123 833-847. (https://doi.org/10.1016/j.cell.2005.09.011)

Krishnamurthy H, Kishi H, Shi M, Galet C, Bhaskaran RS, Hirakawa T \& Ascoli M 2003 Postendocytotic trafficking of the follicle-stimulating hormone (FSH)-FSH receptor complex. Molecular Endocrinology 17 2162-2176. (https://doi.org/10.1210/me.2003-0118)

la Marca A, Morgante G, Palumbo M, Cianci A, Petraglia F \& De Leo V 2002 Insulin-lowering treatment reduces aromatase activity in response to follicle-stimulating hormone in women with polycystic ovary syndrome. Fertility and Sterility 78 1234-1239. (https://doi.org/10.1016/ S0015-0282(02)04346-7)

LaPolt PS, Tilly JL, Aihara T, Nishimori K \& Hsueh AJ 1992 Gonadotropininduced up- and down-regulation of ovarian follicle-stimulating hormone $(\mathrm{FSH})$ receptor gene expression in immature rats: effects of pregnant mare's serum gonadotropin, human chorionic gonadotropin, and recombinant FSH. Endocrinology 130 1289-1295. (https://doi. org/10.1210/endo.130.3.1537292)

Marlin MC \& Li G 2015 Differential effects of overexpression of Rab5 and Rab22 on autophagy in PC12 cells with or without NGF. Methods in Molecular Biology 1298 295-304. (https://doi.org/10.1007/978-1-49392569-8_25)

Mastorakos G, Lambrinoudaki I \& Creatsas G 2006 Polycystic ovary syndrome in adolescents: current and future treatment options. Paediatric Drugs 8 311-318. (https://doi.org/10.2165/00148581-20060805000004)

Mendelson CR \& Kamat A 2007 Mechanisms in the regulation of aromatase in developing ovary and placenta. Journal of Steroid Biochemistry and Molecular Biology 106 62-70. (https://doi.org/10.1016/j. jsbmb.2007.05.001)

Menon KM \& Menon B 2012 Structure, function and regulation of gonadotropin receptors - a perspective. Molecular and Cellular Endocrinology 356 88-97. (https://doi.org/10.1016/j.mce.2012.01.021)

Miaczynska M, Christoforidis S, Giner A, Shevchenko A, UttenweilerJoseph S, Habermann B, Wilm M, Parton RG \& Zerial M 2004 APPL proteins link Rab5 to nuclear signal transduction via an endosomal compartment. Cell 116 445-456. (https://doi.org/10.1016/S00928674(04)00117-5)

Nielsen E, Christoforidis S, Uttenweiler-Joseph S, Miaczynska M, Dewitte F, Wilm M, Hoflack B \& Zerial M 2000 Rabenosyn-5, a novel Rab5 effector, is complexed with hVPS45 and recruited to endosomes through a FYVE finger domain. Journal of Cell Biology 151 601-612. (https://doi.org/10.1083/jcb.151.3.601)

Nishi Y, Yanase T, Mu Y, Oba K, Ichino I, Saito M, Nomura M, Mukasa C, Okabe T, Goto K et al. 2001 Establishment and characterization of a steroidogenic human granulosa-like tumor cell line, KGN, that expresses functional follicle-stimulating hormone receptor. Endocrinology 142 437-445. (https://doi.org/10.1210/endo.142.1.7862)

Olchowik M \& Miaczyńska M 2009 Effectors of GTPase Rab5 in endocytosis and signal transduction. Postepy Biochemii 55 171-180.

Pereira-Leal JB \& Seabra MC 2001 Evolution of the Rab family of small GTP-binding proteins. Journal of Molecular Biology 313 889-901. (https://doi.org/10.1006/jmbi.2001.5072)

Richards JS 1980 Maturation of ovarian follicles: actions and interactions of pituitary and ovarian hormones on follicular cell differentiation. Physiological Reviews $60 \quad 51-89 . \quad$ (https://doi.org/10.1152/ physrev.1980.60.1.51)

Richards JS, Jonassen JA, Rolfes AI, Kersey K \& Reichert LE Jr 1979 Adenosine 3',5'-monophosphate, luteinizing hormone receptor, and progesterone during granulosa cell differentiation: effects of estradiol and follicle-stimulating hormone. Endocrinology 104 765-773. (https:// doi.org/10.1210/endo-104-3-765)

Schwartz SL, Cao C, Pylypenko O, Rak A \& Wandinger-Ness A 2007 Rab GTPases at a glance. Journal of Cell Science 120 3905-3910. (https://doi. org/10.1242/jcs.015909)

Segretain D, Gilleron J, Carette D, Denizot JP \& Pointis G 2010 Differential time course of FSH/FSH receptor complex endocytosis within Sertoli and germ cells during rat testis development. Developmental Dynamics 239 1113-1123. (https://doi.org/10.1002/dvdy.22261)

Shi Y, Zhao H, Shi Y, Cao Y, Yang D, Li Z, Zhang B, Liang X, Li T, Chen J et al. 2012 Genome-wide association study identifies eight new risk loci for polycystic ovary syndrome. Nature Genetics 44 1020-1025. (https:// doi.org/10.1038/ng.2384)

Simoni M, Gromoll J \& Nieschlag E 1997 The follicle-stimulating hormone receptor: biochemistry, molecular biology, physiology, and pathophysiology. Endocrine Reviews 18 739-773. (https://doi. org/10.1210/edrv.18.6.0320) 
Sorkin A \& Von Zastrow M 2002 Signal transduction and endocytosis: close encounters of many kinds. Nature Reviews. Molecular Cell Biology 3 600-614. (https://doi.org/10.1038/nrm883)

Stenmark H 2009 Rab GTPases as coordinators of vesicle traffic. Nature Reviews: Molecular Cell Biology 10 513-525. (https://doi.org/10.1038/ nrm2728)

Stenmark H, Vitale G, Ullrich O \& Zerial M 1995 Rabaptin-5 is a direct effector of the small GTPase Rab5 in endocytic membrane fusion. Cell $\mathbf{8 3}$ 423-432. (https://doi.org/10.1016/0092-8674(95)90120-5)

Su X, Lodhi IJ, Saltiel AR \& StahI PD 2006 Insulin-stimulated interaction between insulin receptor substrate 1 and p85alpha and activation of protein kinase B/Akt require Rab5. Journal of Biological Chemistry 281 27982-27990. (https://doi.org/10.1074/jbc.M602873200)

Su WC, Chao TC, Huang YL, Weng SC, Jeng KS \& Lai MM 2011 Rab5 and class III phosphoinositide 3-kinase Vps34 are involved in hepatitis C virus NS4B-induced autophagy. Journal of Virology 85 10561-10571. (https://doi.org/10.1128/JVI.00173-11)

Walker WH, Fucci L \& Habener JF 1995 Expression of the gene encoding transcription factor cyclic adenosine $3^{\prime}, 5^{\prime}$-monophosphate (cAMP) response element-binding protein (CREB): regulation by folliclestimulating hormone-induced CAMP signaling in primary rat Sertoli cells. Endocrinology 136 3534-3545. (https://doi.org/10.1210/ endo.136.8.7628390)

Zhang B, Shozu M, Okada M, Ishikawa H, Kasai T, Murakami K, Nomura K, Harada N \& Inoue M 2010 Insulin-like growth factor I enhances the expression of aromatase P450 by inhibiting autophagy. Endocrinology 151 4949-4958. (https://doi.org/10.1210/en.2010-0294)

Received 8 January 2018

First decision 16 January 2018

Revised manuscript received 1 April 2018

Accepted 6 April 2018 